\title{
Effect of colchicine treatment on primary knee osteoarthritic patients
}

\begin{abstract}
Background and Aim: It was hypothesized that cartilage degradation in osteoarthritis (OA) facilitates monosodium urate crystals nucleation which by its turn contribute to progression of OA. The aim of the current study is to assess the efficacy of oral colchicine treatment in patients having primary Knee OA (KOA).
\end{abstract}

Patients and Methods: This clinical trial included 40 patients with KOA. After baseline evaluation, patients received oral colchicine $0.5 \mathrm{mg}$ twice daily for 16 -weeks period. The outcome measures (knee pain and WOMAC scale) and human urinary C-terminal telopeptide of type II collagen (UCTX-II) were evaluated at inclusion and at the end of study.

Results: Colchicine had significantly improved frequency of knee effusion (from $62.5 \%$ to $40.0 \%$, $\mathrm{p}=0.044$ ), tenderness score (from $1.9 \pm 0.8$ to $1.3 \pm 0.6, \mathrm{p}<0.001$ ), VAS-pain score (from $60.2 \pm 11.7$ to $48.6 \pm 11.5, p<0.001$ ) and the total WOMAC score (from $48.2 \pm 6.4$ to $42.8 \pm 9.2, p=0.003$ ). The uCTX-II was reduced from $447.4 \pm 63.4 \mathrm{ng} / \mathrm{mmol}$ at baseline evaluation to $428.9 \pm 106.7 \mathrm{ng} / \mathrm{mmol}$ at the end of treatment but this difference was not significant. Only one patient complained of gastrointestinal side effect in the form of diarrhea.

Conclusion: Colchicine appears to be an effective and safe option treatment in primary KOA. Colchicine reduced pain and stiffness and improves functionality with good safety profile

Keywords: Colchicine • primary knee osteoarthritis • WOMAC, CTX-II

\section{Introduction}

Knee osteoarthritis (KOA) is a leading cause of disability in elderly [1]. More than $80 \%$ of KOA patients had movement limitation, $25 \%$ had limitation in activities of daily living, $14 \%$ require aid with routine needs while $11 \%$ require help with personal care $[2,3]$. The global prevalence of $\mathrm{KOA}$ is expected to be duplicated in the next decade due to the increase in the overall lifespan and increase in obesity levels $[4,5]$.

Despite the high prevalence and the significant associated disability, most present treatment paradigms are limited to palliative measures largely focus on analgesia and in severe conditions joint replacement is conducted. Many agents have demonstrated structural modifying capabilities [6]; however, clinical trials did not find promising results regarding the side effects, satisfactory symptomatic relief and did not demonstrate structural modifying endpoints as defined by the Food and Drug Administration (FDA) [7].

The relationship between uric acid and osteoarthritis (OA) had long been observed, and the pathological link of gout with OA had been postulated [8], albeit that this association was found insignificant after adjustment for the confound- ing risk factors such as Body Mass Index (BMI) $[9,10]$.

In patients with OA without clinical gout, synovial fluid uric level is strongly associated with interleukin (IL)-18, IL-1 $\beta$ and radiological severity of OA [11]. Acute attacks of gout may co-exist in a joint with clinical OA implying that $\mathrm{OA}$ might predispose to local nucleation of monosodium urate (MSU) crystals [12]. MSU crystal deposition result in inhibition of chondrocyte viability and function [13]. It was hypothesized that in OA the cartilage degradation may facilitate the deposition of MSU crystals, a process that contributes to progression of OA [14]. This concept of the pathogenesis of $\mathrm{OA}$ provide a rationale that the established treatment for gout, colchicine, can be a strong candidate as an effective treatment for OA.

However, the results of trials that evaluated the effect of colchicine on KOA symptoms were inconsistent. Many clinical trials had reported the pain relieving and symptoms improving effects of colchicine in KOA [15-17]. However, in the recent study of Leung et al. colchicine did not reduce KOA symptoms over a 16-week study period [18]. The aim of the current study is to assess the efficacy of oral colchicine in patients with primary KOA.
Tamer omar El said*1, Mai

A Elkosaier', Ibrahim A Elboghdady' \& Mohammed M Elarman ${ }^{2}$

'Department of Rheumatology and Rehabilitation, Mansoura University Faculty of Medicine, Mansoura, Egypt ${ }^{2}$ Department of Clinical Pathology, Mansoura University Faculty of Medicine, Mansoura, Egypt

*Author for correspondence: roberto. tamer-rheum@hotmail.com 


\section{Methods}

\section{Patients}

We invited 40 consecutive patients with primary KOA to participate in this clinical trial. Patients were recruited during the period from September 2016 to March 2017, from the outpatient clinic, Rheumatology and Rehabilitation Department, Mansoura University Hospital. Prior inclusion in the study, the purpose and procedures of the research were discussed with the patients. All patients provided an informed written consent to participate.

\section{Inclusion and exclusion criteria}

Study inclusion required fulfilling the clinical and radiographic American College of Rheumatology (ACR) criteria for KOA [19] with Kellgrene-Lawrence (KL) grade 2 or 3 in at least one knee radiography [20]. Patients with other joint diseases, inflammatory arthritides, past history of gout or podagra, hyperuricemia, chondrocalcinosis, KOA radiographic KL grade of 1 or 4 , knee trauma, knee arthroscopy, either knee arthroplasty, decide arthroplasty in next 16-weeks period, significant renal, hepatic, muscular or cognitive impairment were excluded from the study. Participants on current treatments that inhibit cytochrome-450 and/ or P-glycoprotein-15 to avoid increased risk of colchicine-induced toxicity.

\section{Clinical assessment}

At baseline, patients were clinically assessed through full history taking followed by general and local examinations as well as review of the medical records. The following data were collected: Personal and demographic data (age and sex), duration of the OA, calculation of BMI, presence of co-morbidities, current medications. In the current study, we examined both knees but only the data of the knee fulfilled entry criteria; or the more symptomatic knee at the baseline was designated as affected knee.

\section{Laboratory investigations}

Blood samples were collected at baseline and at end of the study. The following biomarkers were assessed: Erythrocyte Sedimentation Rate (ESR), C-Reactive Protein (CRP) and serum uric acid. Enzyme-linked immunosorbent assay (ELISA) was utilized to determine the human urinary C-terminal telopeptide of type II collagen (uCTX-II) level in urine. Morning urine (5$10 \mathrm{~mL}$ ) was collected and was centrifuged at 2500 $\mathrm{rpm} / \mathrm{min}$ for 20 minutes. The supernatant was transferred to centrifuge tube and kept at $-80^{\circ} \mathrm{C}$. After dissolution, the samples were diluted five times. The diluted samples and standard sample $(100 \mu \mathrm{L})$ were added into coated wells and incubated at $37^{\circ}$ for one hour. The plate was washed five times, $50 \mu \mathrm{L}$ enzyme was added into every well and incubated at $37^{\circ}$ for one hour. Except for blank well, $50 \mu \mathrm{L}$ color-developing agents $\mathrm{A}$ and $\mathrm{B}$ were added into each well for 15 minutes in dark place. Stop solution was used to stop the reaction. The optical density (OD) value at $450 \mathrm{~nm}$ was recorded.

\section{Radiological assessment}

Bilateral antero-posterior weight-bearing knee radiographs were obtained. Knee radiographs were evaluated with the KL grading scale [20].

\section{Intervention}

Patients received oral colchicine $0.5 \mathrm{mg}$ twice per day for 16-weeks period. All patients were forbidden to continue their existing topical analgesic as well as nonsteroidal antiinflammatory drugs if any. There was a washout period of 7 days. Paracetamol $\leq 3 \mathrm{~g} /$ day as rescue analgesic was allowed, and paracetamol pills count was recorded at end of the study. During the study duration, patients were instructed not to start any new treatments for the $\mathrm{KOA}$, including acupuncture, exercises or physical therapy.

\section{Outcomes}

The following outcome measures were evaluated at baseline and at the end of the study: Knee pain (measured using the Visual Analogue Scale (VAS) [21]) and Western Ontario and McMaster Universities OA Index (WOMAC, Likert scale) [22].

\section{Statistical analysis}

All statistical analyses were done by using SPSS v. 20.0. Variables categorical data was presented in number and percent. Chi-square test was used to compare variables containing categorical data. Variable with continuous data were presented in mean \pm Standard Deviation (SD). Student t-test was used for comparison between the two variables containing continuous data. The paired t-test was used to compare within the same group. Significance was set at p value $<0.05$.

\section{Results}

This study included 40 patients with KOA; 34 (86.0\%) females and $6(14.0 \%)$ males. The age of the patients ranged from 50 to 73 years with an average of $55.9 \pm 5.0$ years. The BMI of the 
patients ranged from 25.3 to $46.9 \mathrm{~kg} / \mathrm{m}^{2}$ with an average of $35.3 \pm 6.4 \mathrm{~kg} / \mathrm{m}^{2}$. The average KOA duration was $16.7 \pm 6.2$ years (ranged from 5.0 to 26.0 years). The average serum uric acid of the patients was $5.5 \pm 0.6 \mathrm{mg} / \mathrm{dl}$ (ranged from 4.4 to $6.5 \mathrm{mg} / \mathrm{dl}$ ) (Table 1).

In this study, all but 4 patients had bilateral KOA. In these 4 patients the affected knee is included in the statistical analysis. In the remaining 36 patients the more affected knee is designated as the affected knee and hence $26(65.0 \%)$ right knees and $14(35.0 \%)$ left knees were studied. The radiological examination of these knees revealed that $15(37.5 \%)$ knees were grade 2 and $25(62.5 \%)$ knees were grade 3 in the KellgrenLawrence grading scale (Table 1).

Table 2 compares the outcome measure and the uCTX-II between the baseline evaluation and after 16 weeks of colchicine treatment. The frequency of knee effusion, tenderness score and VAS-pain score was significantly improved after colchicine treatment. The total WOMAC score as well as WOMAC subscales were also significantly improved after 16 weeks of colchicine treatment. On the other hand, the uCTX-II level did not differ significantly after colchicine treatment in comparison to the baseline value (Table 2).

\section{Discussion}

Results of the present study showed that the frequency of knee effusion, tenderness score and VAS-pain score was significantly improved after colchicine treatment. Moreover, the total

Table 1. Personal data and medical history of the patients.

\begin{tabular}{|c|c|}
\hline \multicolumn{2}{|c|}{ Age (years) } \\
\hline Range & $50-73$ \\
\hline Mean \pm SD & $55.9 \pm 5.0$ \\
\hline Female sex (n, \%) & $34,86.0 \%$ \\
\hline \multicolumn{2}{|c|}{ BMI (kg/m ${ }^{2}$ ) } \\
\hline Range & $25.3-46.9$ \\
\hline Mean \pm SD & $35.3 \pm 6.4$ \\
\hline Duration of KOA (years) \\
\hline Range & $5.0-26.0$ \\
\hline Mean \pm SD & $16.7 \pm 6.2$ \\
\hline \multicolumn{2}{|c|}{ Studied knee } \\
\hline Right & $26,65.0$ \\
\hline Left & $14,35.0$ \\
\hline Radiographic Kellgren-Lawrence grade \\
\hline Grade 2 & $15,37.5 \%$ \\
\hline Grade 3 & $25,62.5 \%$ \\
\hline Serum uric acid (mg/dl) \\
\hline Range & $4.4-6.5$ \\
\hline Mean \pm SD & $5.5 \pm 0.6$ \\
\hline
\end{tabular}

WOMAC score as well as WOMAC subscales were also significantly improved after 16 weeks of colchicine treatment. On the other hand, the uCTX-II level did not differ significantly after colchicine treatment in comparison to the baseline value. Interestingly, only one patient complained of gastrointestinal side effect in the form of diarrhea.

The effectiveness of colchicine in KOA had been reported by former small clinical trials, although these studies had some limitations. In the study of Das et al. 39 adult females and males with their age ranged from 40 to 75 years with KOA diagnosed according to the ACR criteria were enrolled. Patients received intraarticular steroid injection and then randomly allocated either to take colchicine $0.5 \mathrm{mg}$ twice daily or placebo over 5 months. In agreement with our results, the study found that colchicine produced significantly greater symptomatic benefit $(30 \%)$ in terms of pain and function of WOMAC scale. However, this study, a subgroup of patients who received intra-articular corticosteroids had significant knee effusions and there was no data regarding the severity of the KOA, thus limiting its generalizability [15].

In another study, Das et al. randomized $36 \mathrm{pa}$ tients with KOA diagnosed according to the ACR criteria with their age ranged from 40 to 75 years. Patients receive colchicine $0.5 \mathrm{mg}$ twice a day or to receive placebo. All patients also received $100 \mathrm{mg}$ nimesulide twice daily for 4 weeks. Also in agreement with our results, KOA patients in colchicine + nimesulide group showed significantly higher improvement rate at 20 -weeks than did the receiving placebo + nimesulide group ( $57.9 \%$ vs $23.5 \%$ in total WOMAC score) [16].

In the study of Aran et al. 61 post-menopausal patients with primary KOA were enrolled. Patient had no evidence of chondrocalcinosis [17]. Participants were randomized into 2 groups to receive $0.5 \mathrm{mg}$ colchicine twice daily or placebo. All patients received common OA treatment. Acetaminophen $<2 \mathrm{gm} /$ day was allowed as rescue-analgesic. The results revealed that, Acetaminophen consumption was significantly lesser in the colchicine in comparison to the placebo. Colchicine group had significantly higher improvement rate at the end of 3-months period in comparison to the placebo in terms of patient's and physician's global assessment measures. These findings were consistent with ours.

Erden et al. had enrolled 60 patients with KOA 
Table 2. Comparison of the knee outcome measures between baseline and after colchicine treatment.

\begin{tabular}{|c|c|c|c|c|c|}
\hline & $\begin{array}{l}\text { At baseline } \\
\text { evaluation }\end{array}$ & $\begin{array}{l}\text { After colchicine } \\
\text { treatment }\end{array}$ & $\begin{array}{c}\text { Mean } \\
\text { difference }\end{array}$ & $95 \%, \mathrm{Cl}$ & $\mathbf{p}$ \\
\hline Effusion & $25,62.5 \%$ & $16,40.0 \%$ & & & 0.044 \\
\hline $\begin{array}{l}\text { Tenderness } \\
\text { score }\end{array}$ & $1.9 \pm 0.8$ & $1.3 \pm 0.6$ & 0.6 & $0.29-0.92$ & $<0.001$ \\
\hline $\begin{array}{l}\text { VAS-pain score } \\
\qquad(\mathrm{mm})\end{array}$ & $60.2 \pm 11.7$ & $48.6 \pm 11.5$ & 11.6 & $6.41-16.79$ & $<0.001$ \\
\hline \multicolumn{6}{|c|}{ WOMAC score } \\
\hline Pain subscale & $11.2 \pm 1.9$ & $9.1 \pm 2.8$ & 2.1 & $1.04-3.17$ & $<0.001$ \\
\hline $\begin{array}{l}\text { Stiffness } \\
\text { subscale }\end{array}$ & $3.4 \pm 0.6$ & $2.7 \pm 0.7$ & 0.7 & $0.41-0.99$ & $<0.001$ \\
\hline $\begin{array}{l}\text { Function } \\
\text { subscale }\end{array}$ & $33.7 \pm 4.7$ & $30.9 \pm 6.2$ & 2.8 & $0.35-5.25$ & 0.03 \\
\hline Total score & $48.2 \pm 6.4$ & $42.8 \pm 9.2$ & 5.4 & $1.87-8.93$ & 0.003 \\
\hline $\begin{array}{l}\text { uCTX-II (ng/ } \\
\text { mmol) }\end{array}$ & $447.4 \pm 63.4$ & $428.9 \pm 106.7$ & 18.5 & $-20.57-57.57$ & 0.35 \\
\hline
\end{tabular}

diagnosed according to the ACR criteria (radiological severity of 2-3). All patients had crystals of calcium pyrophosphate. Patients randomized were into two groups: colchicine $1.5 \mathrm{mg}+$ acetaminophen $3 \mathrm{~g}$ per day versus acetaminophen 3 $\mathrm{g}$ per day. The patients were followed up for 6 months. The results of the study had shown that the combination of colchicine and paracetamol resulted in significantly better improvement of WOMAC score than paracetamol alone [23].

Colchicine has various anti-inflammatory actions. Colchicine preferentially accumulates in leucocytes such that its concentration in neutrophils reach greater than 16-fold the peak plasma level. This high intra-cellular concentration may lead to suppressed neutrophil recruitment, suppressed MSU crystals-enhanced inflammasome and suppressed IL- $1 \beta$ synthesis [5]. In addition, colchicine may suppress inflammation throughout anti-oxidant effect related to supression of superoxide anion [24].

Conversely, Leung et al. randomly assigned $109 \mathrm{KOA}$ patients into two groups to compare 16 -weeks period of $0.5 \mathrm{mg}$ twice-daily oral colchicine versus placebo. The study reported that colchicine reduced inflammation (serum high sensitivity CRP and synovial fluid IL-6, 8 and 18 and tumor necrosis factor- $\alpha$ ) and high bone turnover biomarkers (synovial fluid CTX-I) correlated with severity and progression of OA, however, the study reported that colchicine was no superior than placebo in improving KOA symptoms after 16-weeks period. Notably, placebo group showed higher (but insignificant) response rate than colchicine group $(49.1 \%$ vs $38.9 \%$ ) [18].
The results of our study had shown that the uCTX-II level did not differ significantly after colchicine treatment in comparison to the baseline value despite the clinical improvement of the symptoms of KOA. The absence of halting progression of $\mathrm{OA}$ process with short-term treatment course may not refute the probability of long-term useful effect on the progression of the KOA and thus, a longer treatment duration is required to translate the biological actions into improvement in KOA outcome.

The findings of our study and the previous clinical trials serve as a basis for future larger long-term multi-centered trials with long-term radiological or MRI follow-up to assess effect of colchicine in slowing or halting progression of the OA structural deterioration.

\section{Conclusion}

Colchicine appears to be an effective and safe option treatment in primary KOA. Colchicine reduced pain and stiffness and improves functionality with good safety profile.

\section{References}

1. Grazio S, Balen D. Obesity: Risk factor and predictor of osteoarthritis. Lijec. Vjesn. 131(1-2), 22-26 (2009).

2. Guccione AA, Felson DT, Anderson JJ et al. The effects of specific medical conditions on the functional limitations of elders in the Framingham Study. Am. J. Public. Health. 84(3), 351-358 (1994).

3. Neogi T. The epidemiology and impact of pain in osteoarthritis. Osteoarthr. Cartil. 21(9), 11451153 (2013).

4. Holt HL, Katz JN, Reichmann WM et al. 
Forecasting the burden of advanced knee osteoarthritis over a 10 -year period in a cohort of 60-64 year-old US adults. Osteoarthr. Cartil. 19(1), 44-50 (2011).

5. Leung YY, Thumboo J, Wong BS et al. Colchicine effectiveness in symptom and inflammation modification in knee osteoarthritis (COLKOA): study protocol for a randomized controlled trial. Trials. 30(16), 200 (2015).

6. Martel-Pelletier J, Wildi LM, Pelletier JP. Future therapeutics for osteoarthritis. Bone. 51(2), 297311 (2012).

7. Conaghan PG, Hunter DJ, Maillefert JF et al. Summary and recommendations of the OARSI FDA osteoarthritis assessment of structural change working group. Osteoarthr. Cartil. 19(5), 606-610 (2011).

8. Roddy E, Doherty M. Gout and osteoarthritis: A pathogenetic link? Joint. Bone. Spine. 79(5), 425-427 (2012).

9. Schouten JS, van den Ouweland FA, Valkenburg HA. A 12 year follow up study in the general population on prognostic factors of cartilage loss in osteoarthritis of the knee. Ann. Rheum. Dis. 51, 932-937 (1992).

10. Ding X, Zeng C, Wei J et al. The associations of serum uric acid level and hyperuricemia with knee osteoarthritis. Rheumatol. Int. 36(4), 567573 (2016).

11. Denoble AE, Huffman KM, Stabler TV et al. Uric acid is a danger signal of increasing risk for osteoarthritis through inflammasome activation. Proc. Natl. Acad. Sci. USA. 108(5), 2088-2093 (2011).

12. Ma CA, Leung YY. Exploring the link between uric acid and osteoarthritis. Fron. Med. 4, 225 (2017).

13. Chhana A, Callon KE, Pool B et al. The effects of monosodium urate monohydrate crystals on chondrocyte viability and function: Implications for development of cartilage damage in gout. $J$. Rheumatol. 40, 2067-2074 (2013).

14. Muehleman C, Li J, Aigner T, Rappoport L et al. Association between crystals and cartilage degeneration in the ankle. J. Rheumatol. 35(6), 1108-1117 (2008).

15. Das SK, Mishra K, Ramakrishnan S et al. A randomized controlled trial to evaluate the slow- acting symptom modifying effects of a regimen containing colchicine in a subset of patients with osteoarthritis of the knee. Osteoarthr. Res. Soc. 10(4), 247-252 (2002).

16. Das SK, Ramakrishnan S, Mishra K et al. A randomized controlled trial to evaluate the slowacting symptom-modifying effects of colchicine in osteoarthritis of the knee: A preliminary report. Arthritis. Rheum. 47(3), 280-284 (2002).

17. Aran S, Malekzadeh S, Seifirad S. A doubleblind randomized controlled trial appraising the symptom-modifying effects of colchicine on osteoarthritis of the knee. Clin. Exp. Rheumatol. 29(3), 513-518 (2011).

18. Leung YY, Haaland B, Huebner JL et al. Colchicine lack of effectiveness in symptom and inflammation modification in knee osteoarthritis (COLKOA): a randomized controlled trial. Osteoarthr. Cartil. 26(5), 631-640 (2018).

19. Altman R, Asch E, Bloch D et al. Development of criteria for the classification and reporting of osteoarthritis. Classification of osteoarthritis of the knee. Diagnostic and Therapeutic Criteria Committee of the American Rheumatism Association. Arthritis. Rheum. 29(8), 1039-1049 (1986).

20. Kellgren JH, Lawrence JS. Radiological assessment of osteoarthrosis. Ann. Rheum. Dis. 16, 494e502 (1957).

21. McCormack HM, Horne DJ, Sheather S. Clinical applications of visual analogue scales: A critical review. Psychol. Med. 18(4), 1007-1019 (1988).

22. Bellamy N, Buchanan WW, Goldsmith $\mathrm{CH}$ et $a l$. Validation study of WOMAC: A health status instrument for measuring clinically important patient relevant outcomes to antirheumatic drug therapy in patients with osteoarthritis of the hip or knee. J. Rheumatol. 15(12), 1833-1840 (1988).

23. Erden M, Ediz L, Ozcan $\mathrm{H}$ et al. Effect of colchicine on total antioxidant capacity, antioxidant enzymes and oxidative stress markers in patients with knee osteoarthritis. Int. J. Clin. Med. 3, 377-382 (2012).

24. Chia EW, Grainger R, Harper JL. Colchicine suppresses neutrophil superoxide production in a murine model of gouty arthritis: A rationale for use of low-dose colchicine. Br. J. Pharmacol. 153, 1288-1295 (2008). 